\title{
肥厚型心肌病的致病分子机制研究进展
}

宋艳瑞 ${ }^{1}$, 刘忠 ${ }^{2}$, 顾淑莲 ${ }^{2}$, 钱丽娟 ${ }^{3}$, 严庆丰 ${ }^{1}$

1. 浙江大学生命科学学院, 杭州 310058 ;

2. 浙江大学医学院附属第一医院, 杭州 310003 ;

3. 浙江省肿瘤医院, 杭州 310022

摘要：肥厚型心肌病(Hypertrophic cardiomyopathy, HCM)是以左心室及室间隔不对称肥厚为基本特征的原发性 心肌病, 其发病率约为 $0.2 \%$, 是青少年和运动员心源性猝死的最常见原因。 HCM 的发病年龄、发病程度和猝 死风险等临床表型具有多样性, 通常呈常染色体显性遗传。目前已报道的 HCM 相关突变超过 900 种, 主要定 位在 $\beta$ 肌球蛋白重链基因、肌球蛋白结合蛋白 $\mathrm{C}$ 基因、心拄肌钻蛋白 $\mathrm{T}$ 基因等 13 个心脏肌节蛋白基因; 另一 方面, 越来越多的研究显示线粒体基因突变与 $\mathrm{HCM}$ 发生相关。文章在简单介绍 HCM 形态学特征及临床表型 的基础上，着重综述了 $\mathrm{HCM}$ 的致病分子机制及其最新研究进展。

关键词：肥厚型心肌病；遗传；突变；核基因；线粒体基因

\section{Advances in the molecular pathogenesis of hypertrophic cardiomyopathy}

\author{
SONG Yan-Rui ${ }^{1}$, LIU Zhong ${ }^{2}$, GU Shu-Lian ${ }^{2}$, QIAN Li-Juan ${ }^{3}$, YAN Qing-Feng ${ }^{1}$ \\ 1. College of Life Sciences, Zhejiang University, Hangzhou 310058, China; \\ 2. The First Affiliated Hospital of Zhejiang University, Hangzhou 310003, China; \\ 3. The Cancer Research Centre of Zhejiang Cancer Hospital, Hangzhou 310022, China
}

\begin{abstract}
Hypertrophic Cardiomyopathy (HCM) is a primary cardiac disorder characterized by asymmetric thickening of the septum and left ventricular wall. HCM affects 1 in 500 individuals in the general population, and it is the most common cause of sudden death in the young and athletes. The clinic phenotype of HCM is highly variable with respect to age at onset, degree of symptoms, and risk of sudden death. HCM is usually inherited as a Mendelian autosomal dominant trait. To date, over 900 mutations have been reported in HCM, which were mainly located in 13 genes encoding cardiac sarcomere protein, e.g., MYH7, MYBPC3, and TnT. In addition, more and more mitochondrial DNA mutations were reported to be associated with the pathogenesis of HCM. Based on the description of the clinical phenotype and morphological characteristics, this review focuses on the research in the molecular pathogenic mechanism of HCM and its recent advances.
\end{abstract}

Keywords: hypertrophic cardiomyopathy; genetics; mutations; nuclear genes; mitochondrial DNA

收稿日期: 2010-10-04; 修回日期: 2010-11-29

基金项目: 国家自然科学基金项目(编号：30971599/C060503)，教育部新世纪优秀人才支持计划项目(编号：NCET-06-0526), 浙江省科技计划 项目(编号：2008C23028)和浙江省新世纪 151 人才工程项目(编号：06-2-008)资助

作者简介: 宋艳瑞, 在读硕士研究生, 专业方向：线粒体疾病机制。E-mail: syr601@126.com

通讯作者:严庆丰, 教授, 博士生导师, 研究方向：医学遗传学。E-mail: qfyan@zju.edu.cn 
1869 年法国病理学家 Liouville 等第一次描述 “室间隔心肌不对称增厚”现象，20 世纪 50 年代 Brock $^{[1]}$ 和 $T e a r e^{[2]}$ 分别阐明心肌肥厚和心脏功能障 碍的关系，肥厚型心肌病 (Hypertrophic cardiomyopathy, HCM)最终被确认为一种器质性心脏疾病, 主要表现为左心室和(或)右心室及室间隔不对称肥 厚、心室腔变小、心室顺应性降低。HCM 可在任何 年龄段发病, 是青少年和运动员心源性猝死(Sudden cardiac death, SCD)的最常见原因 ${ }^{[3]}$ 。HCM 在世界范 围内的人群发病率约为 $0.2 \%{ }^{[4]}$, 患者年死亡率约为 $1 \%{ }^{[5]}$ 。

$\mathrm{HCM}$ 具有发病年龄、发病程度和猝死风险等临 床表型多样性的特点, 在深入阐明其病理生理的基 础上, 进一步探究疾病的分子机制, 开展基因诊断 和基因治疗已成为 HCM 新的研究热点和研究方向。 1990 年 Geisterfer-Lowrance 等 ${ }^{[6]}$ 首次报导 $\beta$ 肌球蛋 白重链基因 $(M Y H 7) R 403 \mathrm{Q}$ 突变是 HCM 的致病突变, 这一发现开启了各国科学家研究 HCM 分子致病机 制的大门。在此后 20 年里, 相继有 13 个致病基因 和 900 多个突变被发现 ${ }^{[7]}$ 。

目前普遍认为 $\mathrm{HCM}$ 是由心脏肌节蛋白基因突 变引起的常染色体显性遗传病 ${ }^{[8,9]}$ 。同时, 线粒体基 因调控肥厚型心肌病表型表达的研究也取得进展。 1991 年 Zeviani 等 ${ }^{[10]}$ 报导线粒体 DNA $t R N A^{\operatorname{Leu}(\mathrm{UUR})}$ 突变与母系遗传性肌病和心肌病相关。随后, Raha 等 ${ }^{[11]}$ 研究表明 mtDNA $t R N A^{G l y}$ T9997C 突变或 $t R N A^{I l e} \mathrm{~A} 4295 \mathrm{G}$ 突变是母系遗传性肥厚型心肌病的 重要原因, 使得对 HCM 致病基因的关注从核基因 拓展到线粒体基因。然而, 携带相同致病性突变的 HCM 患者往往具有不同的表型, 核基因背景的不同 可能是导致这一现象的重要原因。 HCM 是致病基 因、修饰基因、环境等因素甚至营养条件等共同作 用的结果。

本文在简单介绍 HCM 形态学特征及临床表型 的基础上, 着重综述 $\mathrm{HCM}$ 的致病分子机制及其最 新研究进展。

\section{HCM 的临床症状和形态学特征}

$\mathrm{HCM}$ 患者在临床上一般表现出呼吸困难、胸 痛、心悸、晕厥及 $\mathrm{SCD}^{[12,13]}$ 。其中, $\mathrm{SCD}$ 是 $\mathrm{HCM}$ 最严重的表型, 往往是 HCM 患者的首次症状表现。
$67 \%$ 的心脏肥厚为不对称型, 主要涉及主动脉下室 间隔 ${ }^{[14]}$ 。右心室肥厚和双心室肥厚的病例较少见。 $\mathrm{HCM}$ 患者心室壁厚度通常在 15 至 $30 \mathrm{~mm}$ 之间, 曾 报道的最厚心室壁厚度为 $60 \mathrm{~mm}^{[14]}$ 。

心肌肥厚主要是心肌细胞肥厚引起, 而非心肌 细胞数目增多 ${ }^{[15]}$ 。组织学研究显示, HCM 患者心肌 细胞肥大，排列紊乱(通常涉及心室的 $20 \%{ }^{[16]}$ )，心 肌间质性纤维化，壁内冠状动脉基质肥厚或异常增

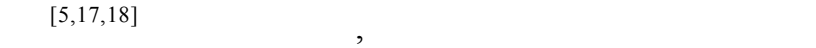
排列的直线束, 但 HCM 患者中, 心肌细胞彼此垂直 或呈不垂直角度杂乱无序的排列, 细胞联接异常, 单个细胞的直径及长度呈现多样性，心肌细胞的细 胞核大小也可能呈现多样性 ${ }^{[15]}$ 。这些表型与舒张期 功能障碍的发生有关, HCM 患者心律不齐的易感性 增加，成为影响发病率和死亡率的重要因素。

\section{$2 \mathrm{HCM}$ 相关的核基因致病突变及其机理}

约 2/3 的 HCM 患者表现出家系遗传的特点, 其 他的为散发性肥厚型心肌病 ${ }^{[19]}$ 。家族性肥厚型心肌 病(Familial hypertrophic cardiomyopathy, FHC)和散 发性肥厚型心肌病具有相同的遗传因素 ${ }^{[20]}$ 。目前普 遍认为 $\mathrm{HCM}$ 是肌节蛋白基因突变引起的常染色体 显性遗传病 ${ }^{[6,21]}$ 。自 1990 年 Geisterfer-Lowrance 等 ${ }^{[6]}$ 第一次报导 $\mathrm{HCM}$ 致病性 $M Y H 7$ 基因 R403Q 突变以 来, 相继发现 13 个致病基因(表 1)的 900 多个突变。 这些致病基因和突变主要位于粗肌丝和细肌丝组分 的编码基因内, $\mathrm{Z}$ 盘不常见。 HCM 也因此被确定为 一种肌节蛋白病。其中 $\beta$ 肌球蛋白重链基因 $(M Y H 7)$ 、 肌球蛋白结合蛋白 $\mathrm{C}$ 基因(MYBPC3)及心脏肌钙蛋 白 $\mathrm{T}$ 基因 $(T n T)$ 是最常见的 3 个 $\mathrm{HCM}$ 致病基因, 由 这些基因突变引起的 HCM 约占 3/4 $4^{[22]}$ 。

\section{1 肌节蛋白基因}

MYH7: 该基因编码的 $\beta$ 肌球蛋白重链( $\beta$-MyHC) 高度保守, 是肌节粗肌丝的重要组成部分, 在心肌 的能量供应产生中具有重要的作用。MYH7 突变引 起的 HCM 占 $35 \% \sim 50 \%$ 。 ${ }^{[23]}$ 。 $M Y H 7$ 突变主要位于肌 球蛋白头部, 可能会影响到肌动蛋白、调节轻链及 ATP 的结合。肌球蛋白杆部和尾部的缺失或插入突 变也有报道 ${ }^{[8]}$, 杆部的突变可能会影响粗肌丝二聚 体的聚合。迄今已报道与 $\mathrm{HCM}$ 相关的 $M Y H 7$ 突变 
表 1 HCM 相关的致病核基因

\begin{tabular}{|c|c|c|c|c|c|}
\hline 基因 & 缩写 & 染色体中位置 & 肌节组分 & 频率 & 参考文献 \\
\hline$\beta$ 肌球蛋白重链基因 & MYH7 & $14 q 12$ & 粗肌丝 & $35 \% \sim 50 \%$ & [23] \\
\hline 肌球蛋白结合蛋白 C & MYBPC 3 & $11 \mathrm{p} 11.2$ & 粗肌丝 & $20 \% \sim 25 \%$ & {$[24]$} \\
\hline 心脏肌钲蛋白 T & $\operatorname{Tn} T 2$ & $1 \mathrm{q} 32$ & 细肌丝 & $\sim 20 \%$ & {$[25]$} \\
\hline 心脏肌钲蛋白 I & $\operatorname{Tn} I 3$ & $19 \mathrm{q} 13.4$ & 细肌丝 & $\sim 5 \%$ & [26] \\
\hline$a$-原肌球蛋白 1 & TPMI & $15 \mathrm{q} 22.1$ & 粗肌丝 & $\sim 5 \%$ & {$[27]$} \\
\hline 肌球轻链蛋白 2 & MYL2 & $12 \mathrm{q} 24.3$ & 粗肌丝 & $1 \sim 2 \%$ & [8] \\
\hline 肌球轻链蛋白 3 & $M Y L 3$ & $3 p 21$ & 粗肌丝 & $<1 \%$ & [8] \\
\hline 心脏肌动蛋白 & $A C T C 1$ & $15 \mathrm{q} 14$ & 细肌丝 & $<5 \%$ & [28] \\
\hline 肌联蛋白 & $T T N$ & $2 \mathrm{q} 31$ & 粗肌丝/Z 盘 & 很少 & [29] \\
\hline 肌肉 LIM 蛋白 & $M L P$ & $11 \mathrm{p} 15.1$ & $\mathrm{Z}$ 盘 & 很少 & {$[30]$} \\
\hline $\mathrm{T}$ 帽蛋白 & $T C A P$ & $17 \mathrm{q} 12$ & $\mathrm{Z}$ 盘 & 很少 & {$[31]$} \\
\hline Myozenin 2 & MYOZ2 & $4 q 26$ & $\mathrm{Z}$ 盘 & 很少 & {$[32]$} \\
\hline 粘着斑蛋白 & $V C L$ & $10 \mathrm{q} 22.1$ & 肌间盘 & 很少 & {$[33]$} \\
\hline
\end{tabular}

超过 180 个 $^{[34]}$ 。MYH7 基因的 $\mathrm{R} 787 \mathrm{H}^{[35]} 、 \mathrm{G} 425 \mathrm{R}^{[36]}$ 突变在不同 $\mathrm{HCM}$ 个体中表现出显著的表型差异, 这表明疾病发生过程中可能还有其他因素如修饰基 因调控致病性突变的表达。

$M Y B P C 3$ ：该基因编码心脏特异性肌球蛋白结 合蛋白, 是脊椎动物横纹肌粗肌丝的重要组成部 分。MYBPC3 基因突变引起的 HCM 占 $20 \sim 25 \%{ }^{[24]}$ 。 MYBPC3 的肌球蛋白结合位点主要位于 $\mathrm{C}$ 末端, 通 过与肌球蛋白及肌动蛋白的结合直接影响心脏肌纤 维的组装 ${ }^{[37]}$ 。位于 $\mathrm{N}$ 末端的调节结构域与肌球蛋白 S2 结构域结合, 通过与 $\beta$ 肌球蛋白相互作用参与 $\mathrm{HCM}$ 的发生 ${ }^{[38]}$ 。MYBPC 3 的 $\mathrm{C} 1$ 及 $\mathrm{C} 2$ 结构域连接 处存在 3 个受 $\beta$ 肾上腺素能调控的磷酸化位点, 参与 心肌的收缩调控 ${ }^{[37]}$ 。目前已鉴定出的 150 多个 ${ }^{[39]}$ $M Y B P C 3$ 突变大部分为插入/缺失突变或剪切位点突 变, 不仅与 $\mathrm{HCM}$ 相关, 还与进行性心脏衰竭、中风 和 SCD 等相关 ${ }^{[40]}$ 。因此 $M Y B P C 3$ 突变携带者同样 需要全面的临床危险性分析。

$T n T$ ：该基因编码的心脏肌钙蛋白 $\mathrm{T}(\mathrm{TnT})$ 是一 种重要的调节蛋白, 与肌钙蛋白 I3(TnI3)和肌钙蛋 白 $\mathrm{C}(\mathrm{TnC})$ 形成复合体, 该复合体与原肌球蛋白 (TPM1) 是钙离子依赖的肌肉收缩过程所必须的 ${ }^{[41]}$ 。 该基因突变引起的 $\mathrm{HCM}$ 约占 $20 \%{ }^{[23]}$ 。

其他与 HCM 相关的编码粗、细肌丝蛋白组分 基因还包括肌钙蛋白 I3 基因(TnI3)、 $\alpha$-原肌球蛋白 基因( TPM1)、肌球轻链蛋白基因( MYL2、MYL3)及心
脏肌动蛋白基因 (ACTC1), 这些基因突变引起的 $\mathrm{HCM}$ 所占比例相对较小 ${ }^{[8,26 \sim 28]}$ 。

\section{$2.2 \mathrm{Z}$ 盘基因、 $\mathrm{VCL}$}

$\mathrm{T}$ 帽蛋白 $(T C A P) 、 \_$Myozenin 2 (MYOZ2)、肌联 蛋白 (TTN) 和肌肉 LIM 蛋白 $(M L P)$ 是 HCM 相关的 $\mathrm{Z}$ 盘基因 ${ }^{[31,32]}$ 。 $\mathrm{Z}$ 盘在肌节收缩与舒张的机械偶联 中具有重要的作用, MYOZ2 和 TCAP 突变可能会改 变蛋白的二级结构, 影响钙离子信号通路及 $\alpha$-辅肌 动蛋白的结合 ${ }^{[31]}$ 。TTN 是已知的人第二大基因, 覆 盖肌节 $\mathrm{Z}$ 盘至 $M$ 线区域，在肌节的组装中扮演重要 的角色。Satoh 等 ${ }^{[29]}$ 发现的 TTN 致病性突变 R740L 可能通过改变 TTN 对 $\alpha$-肌动蛋白的亲和力导致 $\mathrm{HCM}_{\text {。 }}$ MLP 是肌细胞分化相关的核调控因子, MLP 与 $\alpha$-辅肌动蛋白及重要的肌肉特异性转录因子相互 作用的结构域的突变会改变其对以肌动蛋白为基础 的细胞骨架的组装的调控 ${ }^{[30]}$ 。虽然 $\mathrm{Z}$ 盘蛋白基因突 变引起的 $\mathrm{HCM}$ 不常见, 但它使人们对 $Z$ 盘蛋白作为 信号分子参与心脏肥厚应答的认识增加。

粘着斑蛋白(VCL)是一种细胞骨架蛋白，负责 将细肌丝定位于肌间盘。 $V C L$ 基因突变可能会改变 体内肌间盘的组装 ${ }^{[33]}$ 。

研究还发现核基因编码的线粒体磷酸载体蛋白 (PiC)Gly72Glu 同质性突变导致 HCM 患者的肌肉线粒 体 ATP 合成障碍 ${ }^{[42]}$ 。肌节蛋白突变可以激活非心肌细 胞的增殖和纤维化, 从而引起 HCM 病理表型 ${ }^{[43]}$ 。 


\section{$3 \mathrm{HCM}$ 相关的线粒体基因突变及其机理}

线粒体疾病是由线粒体基因或核基因突变导致 氧化磷酸化复合体或膜转运蛋白障碍, 使 ATP 产量 降低的一类临床异常。线粒体疾病患者约 $25 \%$ $40 \%$ 临床表现为心肌病 ${ }^{[44]}$, 肥厚型心肌病是线粒体细胞 病在心脏的主要表现。

已发现的大部分氧化磷酸化缺陷是由线粒体基 因突变引起的 ${ }^{[45]}$ 。Taylor 等 ${ }^{[46]}$ 在两个母系遗传的 HCM 家系中发现两个家系的先证者均携带相同的 mtDNA $t R N A^{I l e} \mathrm{~A} 4300 \mathrm{G}$ 同质突变, 该突变影响线粒体 $t R N A^{I l e}$ 二级结构高度保守区域的一个碱基配对, 导 致心脏组织中成熟的线粒体 $t R N A^{I l e}$ 水平显著下降。 生化分析结果显示 HCM 患者的心肌线粒体电子传 递链复合体 I 和 IV 的活性显著降低, 复合体 II 的活 性正常, 且所有呼吸链组分的活性在骨骼肌及培养 的上皮细胞中完全正常。同时心肌细胞 COX 活性大 大降低, 并且左心室 COX 阴性的细胞多于右心室, 可能是因为左心室对能量的需求远高于右心室 所致。

目前已发现许多参与心肌病发生的 mtDNA 点 突变(表 2)。为了排除核基因背景的影响, Jonckheere 等 ${ }^{[55]}$ 应用胞质杂交技术对携带有线粒体 $A T P 8$ 基因
$\mathrm{T} 8529 \mathrm{C}$ 突变的肥厚型心肌病患者进行研究, 发现氧 化磷酸化复合物 $V$ 活性下降, 导致呼吸链功能障 碍。此外, 缺失突变也是 HCM 发生的重要原因, 国 内也有多家实验室开展了这方面研究。心肌线粒体 重要基因的大片段缺失突变会导致 OXPHOS 障碍, ATP 生成减少, 从而使心肌细胞活动所需能量供应 不足 ${ }^{[63 \sim 65]}$ 。Yi 等 ${ }^{[64]}$ 发现 HCM 患者心肌 mtDNA 的 5 $\mathrm{kb}$ 缺失是其发病的重要原因。但是, 呼吸链功能障 碍导致肥厚型心肌病的致病机理还不明确, 需要进 一步研究证实 ${ }^{[66]}$ 。

线粒体基因组缺乏组蛋白保护，且其修复机制 欠缺, 较高的氧化压力下易发生突变 ${ }^{[67]}$ 。研究发现, 携带 mtDNA 3243 突变的 HCM 患者的心内膜心肌组 织的 ROS 产量增加，血红素氧化酶-1(HO-1)表达降 低 ${ }^{[68]}$ 。高氧化压力促进了线粒体基因组的点突变和 缺失突变, 形成一个恶性循环。因此, 阐明线粒体基 因突变的致病机理尤为重要。

最近, 心肌蛋白对 ATP 的低效利用及由此引起 的 ATP 消耗增加的“能量补偿效应”被认为是 HCM 的主要缺陷。心肌能量供应关键基因如 $C D 36^{[69]}$, 线 粒体 $\mathrm{tRNA}^{[58]}$ 缺陷引起类似于 $\mathrm{HCM}$ 的心脏肥厚表 型支持了这一观点。并且, 能量代谢异常可能是 $\mathrm{HCM}$ 疾病发生过程中的一个早期事件 ${ }^{[70]}$ 。

表 2 肥厚型心肌病相关的 mtDNA 突变

\begin{tabular}{|c|c|c|c|c|c|}
\hline 突变位点 & 基因 & 核苷酸改变 & 氨基酸改变 & 异质性/同质性 & 参考文献 \\
\hline 3243 & $t R N A^{\text {Leu }}$ & $\mathrm{A} \rightarrow \mathrm{G}$ & & 异质 & {$[47,48]$} \\
\hline 3310 & $N D 1$ & $\mathrm{C} \rightarrow \mathrm{T}$ & $\mathrm{P} \rightarrow \mathrm{S}$ & 异质 & [49] \\
\hline 3398 & $N D 1$ & $\mathrm{~T} \rightarrow \mathrm{C}$ & $\mathrm{M} \rightarrow \mathrm{T}$ & 异质 & $\mathrm{http} / / \mathrm{www}$.mitmap.org,2010 \\
\hline 4295 & $t R N A^{\text {Ile }}$ & $\mathrm{A} \rightarrow \mathrm{G}$ & & 异质 & {$[50]$} \\
\hline 4300 & $t R N A^{I l e}$ & $\mathrm{~A} \rightarrow \mathrm{G}$ & & 同质 & [46] \\
\hline 4320 & $t R N A^{I l e}$ & $\mathrm{C} \rightarrow \mathrm{T}$ & & 异质 & [51] \\
\hline 5545 & $t R N A^{T r p}$ & $\mathrm{C} \rightarrow \mathrm{T}$ & & 异质 & http//www.mitmap.org,2010 \\
\hline 8296 & $t R N A^{L y s}$ & $\mathrm{~A} \rightarrow \mathrm{G}$ & & 异质 & [52] \\
\hline 8348 & $t R N A^{L y s}$ & $\mathrm{~A} \rightarrow \mathrm{G}$ & & 异质 & [53] \\
\hline 8363 & $t R N A^{L y s}$ & $\mathrm{G} \rightarrow \mathrm{A}$ & & 异质 & [54] \\
\hline 8529 & ATP8 & $\mathrm{G} \rightarrow \mathrm{A}$ & $\mathrm{W} \rightarrow \mathrm{X}$ & 同质 & [55] \\
\hline 8993 & ATP6 & $\mathrm{T} \rightarrow \mathrm{G}$ & $\mathrm{L} \rightarrow \mathrm{R}$ & 异质 & [56] \\
\hline 9495 & COIII & $\mathrm{T} \rightarrow \mathrm{C}$ & $\mathrm{F} \rightarrow \mathrm{L}$ & 异质 & [57] \\
\hline 9997 & $t R N A^{G l y}$ & $\mathrm{~T} \rightarrow \mathrm{C}$ & & 异质 & [58] \\
\hline 12192 & $t R N A^{H i s}$ & $\mathrm{G} \rightarrow \mathrm{A}$ & & 同质 & [59] \\
\hline 13513 & ND5 & $\mathrm{G} \rightarrow \mathrm{A}$ & $\mathrm{D} \rightarrow \mathrm{N}$ & 异质 & [60] \\
\hline 15243 & Cytb & $\mathrm{G} \rightarrow \mathrm{A}$ & $\mathrm{G} \rightarrow \mathrm{E}$ & 异质 & [61] \\
\hline 15498 & Cytb & $\mathrm{G} \rightarrow \mathrm{A}$ & $\mathrm{G} \rightarrow \mathrm{D}$ & 异质 & [62] \\
\hline
\end{tabular}




\section{$4 \mathrm{HCM}$ 相关的修饰基因突变及其机理}

修饰基因是指除了致病基因外，影响患病个体 表型表达的遗传因素，通常指患者的基因背景 ${ }^{[12]}$ 。 $\mathrm{HCM}$ 不但在遗传上呈现出异质性, 其临床表现也多 种多样。同一患病家系中, 携带相同致病基因突变 的不同成员的心肌肥厚、心力衰竭及 SCD 风险性呈 现出显著差异, 这在一定程度上证明了修饰基因参 与 $\mathrm{HCM}$ 的表型表达。修饰基因本身不足以导致疾 病的发生, 甚至不是疾病发生所必需, 但它可以影 响疾病表型的表达。目前关于 HCM 修饰基因及其 作用还没有系统的研究, 仅仅局限于简单的多态性, 即探索功能性单核甘酸多态性 (Single nucleotide polymorphism, SNPs)和 HCM 发病过程及表型的严 重性之间的关系。据统计, 人基因组包含 30000 到

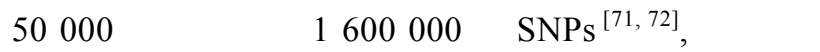
因有多个 SNPs 调控或影响该基因功能。例如, 血管 紧张素 I 转化酶 $(A C E-1)$ 至少有 13 个 $\mathrm{SNPs}{ }^{[73]}$ 。

ACE-1 是一种跨膜蛋白, 它催化血管紧缩素- I 转化为血管紧张素 II, 其中血管紧张素 II 是一种心 脏营养因子。Lechin 等 ${ }^{[74]}$ 在 183 个 HCM 患者中检 测了 ACE-1 功能突变体与左心室肥厚的相关性。发 现 $A C E-1$ 的 3 种基因型 $(D D 、 I D 、 I I)$ 分别存在于 60 、 90 及 33 个 HCM 患者中, 回归分析显示其基因型对 $\mathrm{HCM}$ 患者左心室体积指数及肥厚程度的影响率分 别为 $3.7 \%$ 及 $6.5 \%$; 在另外一个含有 26 个患者的家 系中, 其影响率分别为 $14.7 \%$ 及 $10.4 \%$ 。上述结果表
明 $A C E-1$ 突变与 HCM 中左心室肥厚的严重性相关, 且这种相关性在不同致病基因引起的 HCM 中具有 显著差异。Lim 等 ${ }^{[75]}$ 用氯沙坦处理 $c T n T-\mathrm{Q} 92$ 转基因 小鼠以阻断血管紧张素 II, 发现氯沙坦可以逆转间 质性纤维化, 此外, 胶原- $1 \alpha$ 及转化生长因子- $\beta 1$ 也 下降了 $50 \%$ 。药物干扰修饰基因参与的特异途径可 以逆转 $\mathrm{HCM}$ 的表型, 这为临床上 $\mathrm{HCM}$ 的药物治疗 提供了新思路。

目前, 已发现多个可能参与 HCM 表型表达的 修饰基因(表 3)。然而, 人基因组 SNPs 数量的巨大 决定了 $\mathrm{HCM}$ 相关的基因多态性研究才刚刚起步, 更多修饰基因的鉴定及其调控 HCM 发生的机理仍 需进一步的研究。

\section{5 结语与展望}

$\mathrm{HCM}$ 是致病基因、修饰基因及环境等因素共同 作用的结果, HCM 的发生可能涉及多个独立的信号 通路。阐明这些特异性的途径是有效治疗每一个 HCM 亚型的前提条件。 HCM 的有效治疗需要针对 参与其发病的特异性途径。遗憾的是, 目前, 临床上 $\mathrm{HCM}$ 的药物治疗基本上是经验性的, 没有直接作用 于 $\mathrm{HCM}$ 的发病过程, 在防止、减弱和逆转 $\mathrm{HCM}$ 或 影响其发生方面并没有表现出显著疗效。随着分子 遗传学的发展, 一些参与 $\mathrm{HCM}$ 发生的修饰基因也 被鉴定出来。针对修饰基因参与的特异性信号通路 的药物可以逆转 HCM 的疾病表型, 这为新药的开 发指明了新的方向 ${ }^{[74]}$ 。

表 3 肥厚型心肌病相关的修饰基因

\begin{tabular}{|c|c|c|c|c|}
\hline 染色体中位置 & 缩写 & 基因 & $\mathrm{HCM}$ 相关多态性 & 参考文献 \\
\hline $11 \mathrm{p} 11.2$ & MYBPC 3 & 肌球蛋白结合蛋白 C & $18443 A / G$ & [76] \\
\hline $6 \mathrm{p} 24.1$ & END1 & 内皮素-1 & $?$ & [77] \\
\hline$?$ & $T N F$ & a 肿瘤坏死因子 & $308 G / A$ & [77] \\
\hline $2 \mathrm{p} 11.2$ & $B O P$ & MYND 结构域包含蛋白 1 & $707 C / 710 T / 761 C$ & [78] \\
\hline $5 \mathrm{p} 15.3$ & $\operatorname{Irx} 4$ & Iroquois 家族同源蛋白 4 & $381 A>G$ & [79] \\
\hline $19 \mathrm{q} 13.2-\mathrm{q} 13.3$ & CALM 3 & 钙调蛋白 & $-34 T>A$ & [80] \\
\hline$?$ & $A C E-1$ & 血管紧张素 | 转换酶 & $D D / I D / I I$ & {$[74]$} \\
\hline $10 \mathrm{p} 13$ & ITGA & $\alpha-8$ 整联蛋白 & $?$ & [75] \\
\hline $10 \mathrm{p} 13$ & $C A R P$ & $\begin{array}{l}\text { 半胱氨酸蛋白水解酶募集结构域 } \\
\text { (CARD)包含蛋白 }\end{array}$ & $?$ & [76] \\
\hline $5 \mathrm{p} 15.3$ & $A T 1 R$ & 血管紧张素 II 1 型受体 & $1166 C$ & [81] \\
\hline $8 \mathrm{q} 21-\mathrm{q} 22$ & $C Y P 11 B 2$ & 醛固酮合成酶基因 & $-344 C / T$ & [82] \\
\hline
\end{tabular}


诱导多功能干细胞 (Induced Pluripotent Stem Cells, IPSCs)也是目前研究的热点。Zhao 等 ${ }^{[83]}$ 发现 将胚胎干细胞(Embryonic stem cells, ESCs)注入 Mst1 转基因小鼠中, 可以阻止 Mst 1 过表达诱导的心肌病 的发生。虽然这种研究目前还处于动物实验阶段, 但我们相信随着 IPSCs 和 ESCs 研究的深入, 它们可 能成为治疗 HCM 的又一新途径。

然而, 心肌病的治愈只能通过基因治疗 ${ }^{[84]}$ 。修 复编码线粒体蛋白的线粒体基因和核基因的方法几 乎都存在。但通过改造人类生殖细胞以修复线粒体 缺陷可能只是在理论上可行, 由于宗教和伦理方面 的考虑，临床应用也许只能停留在某一阶段。不过， 我们相信, 随着 HCM 分子机制的阐明及人类传统 观念的改变, 我们会找到缓解甚至治愈 $\mathrm{HCM}$ 的有 效方法。

\section{参考文献(References):}

[1] Brock R. Functional obstruction of the left ventricle (acquired aortic subvalvular stenosis). Guys Hosp Rep, 1957, 106(4): 221-238.

[2] Teare D. Asymmetrical hypertrophy of the heart in young adults. Br Heart J, 1958, 20(1): 1-8.

[3] Maron BJ, Shirani J, Poliac LC, Mathenge R, Roberts WC, Mueller FO. Sudden death in young competitive athletes: Clinical, demographic, and pathological profiles. JAMA, 1996, 276(3): 199-204.

[4] Maron BJ, Towbin JA, Thiene G, Antzelevitch C, Corrado D, Arnett D, Moss AJ, Seidman CE, Young JB. Contemporary definitions and classification of the cardiomyopathies: an American Heart Association Scientific Statement from the Council on Clinical Cardiology, Heart Failure and Transplantation Committee; Quality of Care and Outcomes Research and Functional Genomics and Translational Biology Interdisciplinary Working Groups; and Council on Epidemiology and Prevention. Circulation, 2006, 113(14): 1807-1816.

[5] Hershberger RE, Cowan J, Morales A, Siegfried JD. Progress with genetic cardiomyopathies: screening, counseling, and testing in dilated, hypertrophic, and arrhythmogenic right ventricular dysplasia/cardiomyopathy. Circ Heart Fail, 2009, 2(3): 253-261.

[6] Geisterfer-Lowrance AAT, Kass S, Tanigawa G, Vosberg HP, McKenna W, Seidman CE, Seidman JG. A molecular basis for familial hypertrophic cardiomyopathy: a $\beta$ cardiac myosin heavy chain gene missense mutation. Cell, 1990, 62(5): 999-1006.
[7] Alcalai R, Seidman JG, Seidman CE. Genetic basis of hypertrophic cardiomyopathy: from bench to the clinics. $J$ Cardiovasc Electr, 2008, 19(1): 104-110.

[8] Richard P, Charron P, Carrier L, Ledeuil C, Cheav T, Pichereau C, Benaiche A, Isnard R, Dubourg O, Burban M, Gueffet JP, Millaire A, Desnos M, Schwartz K, Hainque B, Komajda M. Hypertrophic cardiomyopathy: distribution of disease genes, spectrum of mutations, and implications for a molecular diagnosis strategy. Circulation, 2003, 107(17): $2227-2232$

[9] Soor GS, Luk A, Ahn E, Abraham JR, Woo A, Ralph-Edwards A, Butany J. Hypertrophic cardiomyopathy: current understanding and treatment objectives. J Clin Pathol, 2009, 62(3): 226-235.

[10] Zeviani M, Gellera C, Antozzi C, Rimoldi M, Morandi L, Villani F, Tiranti V, DiDonato S. Maternally inherited myopathy and cardiomyopathy: association with mutation in mitochondrial DNA tRNA ${ }^{\text {Leu(UUR) }}$. Lancet, 1991, 338(8760): 143-147.

[11] Raha S, Merante F, Shoubridge E, Myint AT, Tein I, Benson L, Johns T, Robinson BH. Repopulation of rho0 cells with mitochondria from a patient with a mitochondrial DNA point mutation in tRNA(Gly) results in respiratory chain dysfunction. Hum Mutat, 1999, 13(3): 245-254.

[12] Maron BJ. Hypertrophic cardiomyopathy: a systematic review. JAMA, 2002, 287(10): 1308-1320.

[13] Bashyam MD, Savithri GR, Kumar MS, Narasimhan C, Nallari P. Molecular genetics of familial hypertrophic cardiomyopathy (FHC). J Hum Genet, 2003, 48(2): 55-64.

[14] Poliac LC, Barron ME, Maron BJ. Hypertrophic cardiomyopathy. Anesthesiology, 2006, 104(1): 183-192.

[15] Tam SK, Gu W, Mahdavi V, Nadal-Ginard B. Cardiac myocyte terminal differentiation. Potential for cardiac regeneration. Ann N Y Acad Sci, 1995, 752: 72-79.

[16] Marian AJ. Contemporary treatment of hypertrophic cardiomyopathy. Tex Heart Inst J, 2009, 36(3): 194-204.

[17] Marian AJ. Hypertrophic cardiomyopathy: from genetics to treatment. Eur J Clin Invest, 2010, 40(4): 360-369.

[18] 齐建光, 杜军保. 线粒体心肌病临床诊断和基因研究进展. 中国实用儿科杂志, 2005, 20(6): 374-376.

[19] Marian AJ, Roberts R. The molecular genetic basis for hypertrophic cardiomyopathy. J Mol Cell Cardiol, 2001, 33(4): 655-670.

[20] Marian AJ. Genetic determinants of cardiac hypertrophy. Curr Opin Cardiol, 2008, 23(3): 199-205.

[21] 李文, 马沛然, 汪翼, 韩秀珍, 丘厚兴. 线粒体 DNA 点突 变与心肌病关系的研究. 山东医药, 1998, 38(7): 3-4.

[22] Morita H, Rehm HL, Menesses A, McDonough B, Roberts AE, Kucherlapati R, Towbin JA, Seidman JG, Seidman CE. Shared genetic causes of cardiac hypertrophy in children and 
adults. N Engl J Med, 2008, 358(18): 1899-1908.

[23] Seidman CE, Seidman JG. Molecular genetic studies of familial hypertrophic cardiomyopathy. Basic Res Cardiol, 1998, 93(Suppl. 3): 13-16.

[24] Niimura H, Bachinski LL, Sangwatanaroj S, Watkins H, Chudley AE, McKenna W, Kristinsson A, Roberts R, Sole M, Maron BJ, Seidman JG, Seidman CE. Mutations in the gene for cardiac myosin-binding protein $\mathrm{C}$ and late-onset familial hypertrophic cardiomyopathy. $N$ Engl J Med, 1998, 338(18): 1248-1257.

[25] Varnava AM, Elliott PM, Baboonian C, Davison F, Davies MJ, McKenna WJ. Hypertrophic cardiomyopathy: histopathological features of sudden death in cardiac troponin $\mathrm{T}$ disease. Circulation, 2001, 104(12): 1380-1384.

[26] Kimura A, Harada H, Park JE, Nishi H, Satoh M, Takahashi M, Hiroi S, Sasaoka T, Ohbuchi N, Nakamura T, Koyanagi T, Hwang TH, Choo JA, Chung KS, Hasegawa A, Nagai R, Okazaki O, Nakamura H, Matsuzaki M, Sakamoto T, Toshima H, Koga Y, Imaizumi T, Sasazuki T. Mutations in the cardiac troponin I gene associated with hypertrophic cardiomyopathy. Nat Genet, 1997, 16(4): 379-382.

[27] Thierfelder L, Watkins H, MacRae C, Lamas R, McKenna W, Vosberg HP, Seidman JG, Seidman CE. $\alpha$-tropomyosin and cardiac troponin $\mathrm{T}$ mutations cause familial hypertrophic cardiomyopathy: a disease of the sarcomere. Cell, 1994, 77(5): 701-712.

[28] Mogensen J, Klausen IC, Pedersen AK, Egeblad H, Bross P, Kruse TA, Gregersen N, Hansen PS, Baandrup U, Børglum AD. $\alpha$-cardiac actin is a novel disease gene in familial hypertrophic cardiomyopathy. J Clin Invest, 1999, 103(10): R39-R43.

[29] Satoh M, Takahashi M, Sakamoto T, Hiroe M, Marumo F, Kimura A. Structural analysis of the titin gene in hypertrophic cardiomyopathy: identification of a novel disease gene. Biochem Biophys Res Commun, 1999, 262(2): 411-417.

[30] Geier C, Perrot A, Özcelik C, Binner P, Counsell D, Hoffmann K, Pilz B, Martiniak Y, Gehmlich K, van der Ven PFM, Fürst DO, Vornwald A, von Hodenberg E, Nürnberg P, Scheffold T, Dietz R, Osterziel KJ. Mutations in the human muscle LIM protein gene in families with hypertrophic cardiomyopathy. Circulation, 2003, 107(10): 1390-1395.

[31] Hayashi T, Arimura T, Itoh-Satoh M, Ueda K, Hohda S, Inagaki N, Takahashi M, Hori H, Yasunami M, Nishi H, Koga Y, Nakamura $\mathrm{H}$, Matsuzaki M, Choi BY, Bae SW, You CW, Han KH, Park JE, Knöll R, Hoshijima M, Chien KR, Kimura A. Tcap gene mutations in hypertrophic cardiomyopathy and dilated cardiomyopathy. J Am Coll Cardiol, 2004, 44(11): 2192-2201.

[32] Osio A, Tan LL, Chen SN, Lombardi R, Nagueh SF, Shete S, Roberts R, Willerson JT, Marian AJ. Myozenin 2 is a novel gene for human hypertrophic cardiomyopathy. Circ Res, 2007, 100(6): 766-768.

[33] Vasile VC, Ommen SR, Edwards WD, Ackerman MJ. A missense mutation in a ubiquitously expressed protein, vinculin, confers susceptibility to hypertrophic cardiomyopathy. Biochem Biophys Res Commun, 2006, 345(3): 998-1003.

[34] Tanjore R, RangaRaju A, Vadapalli S, Remersu S, Narsimhan $\mathrm{C}$, Nallari $\mathrm{P}$. Genetic variations of $\beta-M Y H 7$ in hypertrophic cardiomyopathy and dilated cardiomyopathy. Indian $J$ Hum Genet, 2010, 16(2): 67-71.

[35] Purushotham G, Madhumohan K, Anwaruddin M, Nagarajaram H, Hariram V, Narasimhan C, Bashyam MD. The MYH7 p.R787H mutation causes hypertrophic cardiomyopathy in two unrelated families. Exp Clin Cardiol, 2010, 15(1): e1-e4.

[36] 王虎, 邹玉宝, 宋雷, 王继征, 孙凯, 宋晓东, 高硕, 张禅 那, 惠汝太. 心脏肌球蛋白重链基因 c. $1273 \mathrm{G} 》 \mathrm{~A}$ 突变与肥 厚型心肌病的关联分析. 遗传, 2009, 31(5): 485-488.

[37] Winegrad S. Cardiac myosin binding protein C. Circ Res, 1999, 84(10): 1117-1126

[38] Vikstrom KL, Leinwand LA. Contractile protein mutations and heart disease. Curr Opin Cell Biol, 1996, 8(1): 97-105.

[39] Oliva-Sandoval MJ, Ruiz-Espejo F, Monserrat L, Hermida-Prieto M, Sabater M, Garcia-Molina E, Ortiz M, Rodriguez-Garcia MI, Núñez L, Gimeno JR, Castro-Beiras A, Valdés M. Insights into genotype-phenotype correlation in hypertrophic cardiomyopathy. Findings from 18 Spanish families with a single mutation in MYBPC3. Heart, 2010, 96(24): 1980-1984

[40] Ehlermann P, Weichenhan D, Zehelein J, Steen H, Pribe R, Zeller R, Lehrke S, Zugck C, Ivandic BT, Katus HA. Adverse events in families with hypertrophic or dilated cardiomyopathy and mutations in the MYBPC3 gene. BMC Med Genet, 2008, 9: 95 .

[41] Perry SV. Troponin T: genetics, properties and function. $J$ Muscle Res Cell Motil, 1998, 19(6): 575-602.

[42] Mayr JA, Merkel O, Kohlwein SD, Gebhardt BR, Böhles H, Fötschl U, Koch J, Jaksch M, Lochmüller H, Horváth R, Freisinger P, Sperl W. Mitochondrial phosphate-carrier deficiency: a novel disorder of oxidative phosphorylation. $\mathrm{Am} \mathrm{J}$ Hum Genet, 2007, 80(3): 478-484.

[43] Teekakirikul P, Eminaga S, Toka O, Alcalai R, Wang LB, Wakimoto H, Nayor M, Konno T, Gorham JM, Wolf CM, Kim JB, Schmitt JP, Molkentin JD, Norris RA, Tager AM, Hoffman SR, Markwald RR, Seidman CE, Seidman JG. Cardiac fibrosis in mice with hypertrophic cardiomyopathy is mediated by non-myocyte proliferation and requires Tgf- $\beta$. $J$ Clin Invest, 2010, 120(10): 3520-3529.

[44] Holmgren D, Wåhlander H, Eriksson BO, Oldfors A, Holme E, Tulinius M. Cardiomyopathy in children with mitochon- 
drial disease; clinical course and cardiological findings. Eur Heart J, 2003, 24(3):280-288.

[45] Marin-Garcia J, Goldenthal MJ. Mitochondrial cardiomyopathy: molecular and biochemical analysis. Pediatr Cardiol, 1997, 18(4): 251-260.

[46] Taylor RW, Giordano C, Davidson MM, d'Amati G, Bain H, Hayes CM, Leonard H, Barron MJ, Casali C, Santorelli FM, Hirano M, Lightowlers RN, DiMauro S, Turnbull DM. A homoplasmic mitochondrial transfer ribonucleic acid mutation as a cause of maternally inherited hypertrophic cardiomyopathy. J Am Coll Cardiol, 2003, 41(10): 1786-1796.

[47] Manouvrier S, Rötig A, Hannebique G, Gheerbrandt JD, Royer-Legrain G, Munnich A, Parent M, Grünfeld JP, Largilliere C, Lombes A. Point mutation of the mitochondrial tRNA(Leu) gene (A $3243 \mathrm{G}$ ) in maternally inherited hypertrophic cardiomyopathy, diabetes mellitus, renal failure, and sensorineural deafness. J Med Genet, 1995, 32(8): 654-656.

[48] 齐建光, 张英, 威豫, 杨艳玲, 无晔, 姜玉武, 秦炣, 杜军 保. 儿童线粒体病心脏损害 23 例临床分析. 实用儿科临 床杂志, 2006, 21(1): 12-13.

[49] Chen J, Hattori Y, Nakajima K, Eizawa T, Ehara T, Koyama M, Hirai T, Fukuda Y, Kinoshita M, Sugiyama A, Hayashi JI, Onaya T, Kobayashi T, Tawata M. Mitochondrial complex I activity is significantly decreased in a patient with maternally inherited type 2 diabetes mellitus and hypertrophic cardiomyopathy associated with mitochondrial DNA C3310T mutation: a cybrid study. Diabetes Res Clin Pract, 2006, 74(2): $148-153$

[50] Merante F, Myint T, Tein I, Benson L, Robinson BH. An additional mitochondrial tRNA ${ }^{\text {Ile }}$ point mutation (A-to-G at nucleotide 4295) causing hypertrophic cardiomyopathy. Hum Mutat, 1996, 8(3): 216-222.

[51] Santorelli FM, Mak SC, Vazquezacevedo M, Gonzalezastiazaran A, Ridaurasanz C, Gonzalezhalphen D, Dimauro S. A novel mitochondrial DNA point mutation associated with mitochondrial encephalocardiomyopathy. Biochem Biophys Res Commun, 1995, 216(3): 835-840.

[52] Akita Y, Koga Y, Iwanaga R, Wada N, Tsubone J, Fukuda S, Nakamura Y, Kato H. Fatal hypertrophic cardiomyopathy associated with an A8296G mutation in the mitochondrial tRNA $^{\text {Lys }}$ gene. Hum Mutat, 2000, 15(4): 382.

[53] Terasaki F, Tanaka M, Kawamura K, Kanzaki Y, Okabe M, Hayashi T, Shimomura H, Ito T, Suwa M, Gong JS, Zhang J, Kitaura Y. A case of cardiomyopathy showing progression from the hypertrophic to the dilated form: association of $\mathrm{Mt} 8348 \mathrm{~A} \rightarrow \mathrm{G}$ mutation in the mitochondrial tRNA(Lys) gene with severe ultrastructural alterations of mitochondria in cardiomyocytes. Jpn Circ J, 2001, 65(7): 691-694.

[54] Santorelli FM, Mak SC, El-Schahawi M, Casali C, Shanske S, Baram TZ, Madrid RE, DiMauro S. Maternally inherited car- diomyopathy and hearing loss associated with a novel mutation in the mitochondrial tRNA ${ }^{\text {Lys }}$ gene (G8363A). Am J Hum Genet, 1996, 58(5): 933-939.

[55] Jonckheere AI, Hogeveen M, Nijtmans LGJ, van den Brand MAM, Janssen AJM, Diepstra JHS, van den Brandt FCA, van den Heuvel LP, Hol FA, Hofste TGJ, Kapusta L, Dillmann U, Shamdeen MG, Smeitink JAM, Rodenburg RJT. A novel mitochondrial ATP8 gene mutation in a patient with apical hypertrophic cardiomyopathy and neuropathy. J Med Genet, 2008, 45(3): 129-133.

[56] Pastores GM, Santorelli FM, Shanske S, Gelb BD, Fyfe B, Wolfe D, Willner JP. Leigh syndrome and hypertrophic cardiomyopathy in an infant with a mitochondrial DNA point mutation (T8993G). Am J Med Genet, 1994, 50(3): 265-271.

[57] Minieri M, Zingarelli M, Shubeita H, Vecchini A, Binaglia L, Carotenuto F, Fantini C, Fiaccavento R, Masuelli L, Coletti A Simonelli L, Modesti A, Di Nardo P. Identification of a new missense mutation in the mtDNA of hereditary hypertrophic, but not dilated cardiomyopathic hamsters. Mol Cell Biochem, 2003, 252(1-2): 73-81.

[58] Merante F, Tein I, Benson L, Robinson BH. Maternally inherited hypertrophic cardiomyopathy due to a novel T-to-C transition at nucleotide 9997 in the mitochondrial tRNA(sup glycine) gene. Am J Hum Genet, 1994, 55(3): 437-446.

[59] Mimaki M, Ikota A, Sato A, Komaki H, Akanuma J, Nonaka I, Goto YI. A double mutation (G11778A and G12192A) in mitochondrial DNA associated with Leber's hereditary optic neuropathy and cardiomyopathy. J Hum Genet, 2003, 48(1): $47-50$.

[60] Wang SB, Weng WC, Lee NC, Hwu WL, Fan PC, Lee WT. Mutation of mitochondrial DNA G13513A presenting with Leigh syndrome, Wolff-Parkinson-White syndrome and cardiomyopathy. Pediatr Neonatol, 2008, 49(4): 145-149.

[61] Valnot I, Kassis J, Chretien D, de Lonlay P, Parfait B, Munnich A, Kachaner J, Rustin P, Rötig A. A mitochondrial cytochrome $b$ mutation but no mutations of nuclearly encoded subunits in ubiquinol cytochrome $c$ reductase (complex III) deficiency. Hum Genet, 1999, 104(6): 460-466.

[62] Obayashi T, Hattori K, Sugiyama S, Tanaka M, Tanaka T, Itoyama S, Deguchi H, Kawamura K, Koga Y, Toshima H, Takeda N, Nagano M, Ito T, Ozawa T. Point mutations in mitochondrial DNA in patients with hypertrophic cardiomyopathy. Am Heart J, 1992, 124(5): 1263-1269.

[63] 富路, 杨继红, 刘徽, 张淑华, 曲秀芬, 吴楠. 扩张型心肌 病与线粒体 DNA 缺失片段的研究. 哈尔滨医科大学学报, 1996, 30(5): 436-438.

[64] 尹桂芝, 张丽容, 李殿富, 张林. 肥厚型心肌病患者心肌 线粒体 DNA 的大片段缺失. 南京铁道医学院学报, 1997, 16(4): 268-270

[65] 尹桂芝, 张丽容, 李殿富, 张丽珊. 肥厚型心肌病患者心 
肌 mtDNA 大片段缺失的探讨. 遗传, 1999, 21(1): 16-18.

[66] 李文, 马沛然, 汪翼, 韩秀珍, 丘厚兴. 线粒体 DNA 点突 变与心肌病关系的研究. 山东医药, 1998, 38(7): 3-4.

[67] 卢建菊, 陆惠玲. 线粒体 DNA 突变与心肌病关系的研究 进展. 法医学杂志, 2001, 17(4): 242-243.

[68] Ishikawa K, Kimura S, Kobayashi A, Sato T, Matsumoto H, Ujiie Y, Nakazato K, Mitsugi M, Maruyama Y. Increased reactive oxygen species and anti-oxidative response in mitochondrial cardiomyopathy. Circ J, 2005, 69(5): 617-620.

[69] Tanaka T, Sohmiya K, Kawamura K. Is CD36 deficiency an etiology of hereditary hypertrophic cardiomyopathy? $\mathrm{J} \mathrm{Mol}$ Cell Cardiol, 1997, 29(1): 121-127.

[70] Luedde M, Flögel U, Knorr M, Grundt C, Hippe HJ, Brors B, Frank D, Haselmann U, Antony C, Voelkers M, Schrader J, Most P, Lemmer B, Katus HA, Frey N. Decreased contractility due to energy deprivation in a transgenic rat model of hypertrophic cardiomyopathy. J Mol Med, 2009, 87(4): 411-422.

[71] Risch NJ. Searching for genetic determinants in the new millennium. Nature, 2000, 405(6788): 847-856.

[72] Sachidanandam R, Weissman D, Schmidt SC, Kakol JM, Stein LD, Marth G, Sherry S, Mullikin JC, Mortimore BJ, Willey DL, Hunt SE, Cole CG, Coggill PC, Rice CM, Ning Z, Rogers J, Bentley DR, Kwok PY, Mardis ER, Yeh RT, Schultz B, Cook L, Davenport R, Dante M, Fulton L, Hillier L, Waterston RH, McPherson JD, Gilman B, Schaffner S, Van Etten WJ, Reich D, Higgins J, Daly MJ, Blumenstiel B, Baldwin J, Stange-Thomann N, Zody MC, Linton L, Lander ES, Altshuler D. A map of human genome sequence variation containing 1.42 million single nucleotide polymorphisms. Nature, 2001, 409(6822): 928-933.

[73] Zhu XF, Bouzekri N, Southam L, Cooper RS, Adeyemo A, McKenzie CA, Luke A, Chen GJ, Elston RC, Ward R. Linkage and association analysis of angiotensin I-converting enzyme (ACE)-gene polymorphisms with ACE concentration and blood pressure. Am J Hum Genet, 2001, 68(5): 1139-1148.

[74] Lechin M, Quiñones MA, Omran A, Hill R, Yu QT, Rakowski H, Wigle D, Liew CC, Sole M, Roberts R,Marian AJ. Angiotensin-I converting enzyme genotypes and left ventricular hypertrophy in patients with hypertrophic cardiomyopathy. Circulation, 1995, 92(7): 1808-1812.

[75] Lim DS, Lutucuta S, Bachireddy P, Youker K, Evans A, Entman M, Roberts R, Marian AJ. Angiotensin II blockade reverses myocardial fibrosis in a transgenic mouse model of human hypertrophic cardiomyopathy. Circulation, 2001, 103(6): 789-791.

[76] Daw EW, Chen SN, Czernuszewicz G, Lombardi R, Lu Y, Ma JZ, Roberts R, Shete S, Marian AJ. Genome-wide mapping of modifier chromosomal loci for human hypertrophic cardiomyopathy. Hum Mol Genet, 2007, 16(20): 2463-2471.

[77] Marian AJ. Modifier genes for hypertrophic cardiomyopathy. Curr Opin Cardiol, 2002, 17(3): 242-252.

[78] Abaci N, Gülec C, Bayrak F, Kömurcu Bayrak E, Kahveci G, Erginel Unaltuna N. The variations of BOP gene in hypertrophic cardiomyopathy. Anadolu Kardiyol Derg, 2010, 10(4): 303-309.

[79] Bayrak F, Kömurcü-Bayrak E, Mutlu B, Kahveci G, Erginel-Ünaltuna N. Genetic analysis of the Irx 4 gene in hypertrophic cardiomyopathy. Turk Kardiyol Dern Ars, 2008, 36(2): 90-95.

[80] Friedrich FW, Bausero P, Sun YL, Treszl A, Krämer E, Juhr D, Richard P, Wegscheider K, Schwartz K, Brito D, Arbustini E, Waldenström A, Isnard R, Komajda M, Eschenhagen T, Carrier L. A new polymorphism in human calmodulin III gene promoter is a potential modifier gene for familial hypertrophic cardiomyopathy. Eur Heart J, 2009, 30(13): 1648-1655.

[81] Coto E, Palacín M, Martín M, Castro MG, Reguero JR, García C, Berrazueta JR, Morís C, Morales B, Ortega F, Corao AI, Díaz M, Tavira B, Alvarez V. Functional polymorphisms in genes of the Angiotensin and Serotonin systems and risk of hypertrophic cardiomyopathy: AT1R as a potential modifier. J Transl Med, 2010, 8: 64.

[82] Kaufman BD, Auerbach S, Reddy S, Manlhiot C, Deng LY, Prakash A, Printz BF, Gruber D, Papavassiliou DP, Hsu DT, Sehnert AJ, Chung WK, Mital S. RAAS gene polymorphisms influence progression of pediatric hypertrophic cardiomyopathy. Hum Genet, 2007, 122(5): 515-523.

[83] Zhao QS, Beck AJ, Vitale JM, Schneider JS, Chang C, Gao SM, Del Re D, Bhaumik M, Yehia G, Sadoshima J, Fraidenraich D. Injection of wild type embryonic stem cells into Mst1 transgenic blastocysts prevents adult-onset cardiomyopathy. Stem Cell Rev, 2011, 7(2): 326-330.

[84] Fosslien E. Mitochondrial medicine--cardiomyopathy caused by defective oxidative phosphorylation. Ann Clin Lab Sci, 2003, 33(4): 371-395. 